\title{
GEORGE DAVID BIRKHOFF AND HIS MATHEMATICAL WORK
}

\author{
MARSTON MORSE
}

The writer first saw Birkhoff in the fall of 1914. The graduate students were meeting the professors of mathematics of Harvard in Sever 20. Maxime Bôcher, with his square beard and squarer shoes, was presiding. In the back of the room, with a different beard but equal dignity, William Fogg Osgood was counseling a student. Dunham Jackson, Gabriel Green, Julian Coolidge and Charles Bouton were in the business of being helpful. The thirty-year-old Birkhoff was in the front row. He seemed tall even when seated, and a friendly smile disarmed a determined face. I had no reason to speak to him, but the impression he made upon me could not be easily forgotten.

His change from Princeton University to Harvard in 1912 was decisive. Although he later had magnificent opportunities to serve as a research professor in institutions other than Harvard he elected to remain in Cambridge for life. He had been an instructor at Wisconsin from 1907 to 1909 and had profited from his contacts with Van Vleck. As a graduate student in Chicago he had known Veblen and he continued this friendsip in the halls of Princeton. Starting college in 1902 at the University of Chicago, he changed to Harvard, remained long enough to get an A.B. degree, and then hurried back to Chicago, where he finished his graduate work in 1907.

It was in 1908 that he married Margaret Elizabeth Grafius. It was clear that Birkhoff depended from the beginning to the end on her deep understanding and encouragement. Her varied talents and charm were reflected in the overflowing hospitality of their home. Their children, Garrett and Barbara (Mrs. Robert Treat Paine, Jr.) are well known to friends of Birkhoff.

Birkhoff admired Moore of Chicago, but not to the point of imitating him. He respected Bôcher no less, and did him the honor next to Poincaré of following his mathematical interests. F. R. Moulton's study of the work of Poincaré had something to do with Birkhoff's own intense reading of Poincaré. Poincaré was Birkhoff's true teacher. There is probably no mathematician alive who has explored the works of Poincaré in full unless it be Hadamard, but in the domains of analysis Birkhoff wholeheartedly took over the techniques and problems of Poincaré and carried on.

Birkhoff's loyalty to Harvard was complete and only occasionally critical. The response of the non-mathematical members of the faculty 
to his early successes was slow at first and he felt this. But he did not wait long before he was recognized by the University as a whole. It is a mark of the intellectual distinction of President Lowell that he hailed Birkhoff as one of Harvard's greatest men and honored him accordingly. He was made Perkins Professor of Mathematics and served at one time as Dean of the Faculty of Arts and Sciences. Birkhoff's colleagues in mathematics at Harvard were as one in their admiration of him and affection for him.

Unlike his colleague Osgood, Birkhoff thought of his contemporaries in Europe, particularly in Germany, as colleagues rather than as teachers. He held Klein lightly, was unenthusiastic over Weierstrass, but gave his full respect to Riemann. Through his papers on non-self-adjoint boundary value problems and asymptotic representations he probably influenced the Hilbert integral equation school as much or as little as it influenced him. His relations with the members of the French and Italian schools of analysis were close, both personally and scientifically. Levi-Civita and Hadamard were among his best friends. Birkhoff was at the same time internationally minded and pro-American. The sturdy individualism of Dickson, E. H. Moore and Birkhoff was representative of American mathematics "coming of age." The work of these great Americans sometimes lacked external sophistication, but it more than made up for this in penetration, power and originality, and justified Birkhoff's appreciation of his countrymen.

Much has been said about Birkhoff's teaching, and each of his pupils will have his tale. There is one thing which may be noted in general: that there is an excellent correlation between the more distinguished Ph.D.'s from Harvard and those who were inspired by Birkhoff's teaching. Among his "tutees" there was an unanalyzed appreciation of Birkhoff's ability to transform a subject into something alive, and a deep gratitude for his painstaking personal effort with them. He never consciously descended to the level of the untutored, nor gave them evidence that he was aware of his own relative elevation. He often improvised in an exposition, thereby illuminating many things besides the immediate theorem. He once said that if his livelihood depended upon it he would vie with those most perfect in exposition. Those who knew him best will agree that he could have done so and successfully. This demonstration however was never demanded.

In his social and political views, he was detached from the world about him and this was sometimes a source of misunderstanding. Proceeding from the dynamic individualism of the Middle West to an environment of tenacious self-sufficiency in New England, he un- 
derstood and interpreted both as conditions for living. His philosophy of life was intellectual rather than emotional. His own conduct of life was extraordinarily well integrated, and free from manifestations of eccentricity. He showed his individualism most clearly in the broad range of suppositions which he could entertain and on which he could vividly speculate. The fact that these speculations were disassociated from his own life was one of the reasons why his extreme detachment was possible. He chose to be brief and simple, but not explanatory. $\mathrm{He}$ could examine a point of view as if it were his very own and later reflect with equal appreciation on an opposite point of view. A hearer who did not understand this range of his speculations could easily receive incorrect impressions of Birkhoff's views. To understand Birkhoff one had to weigh and average his conclusions over a long range of circumstances. Birkhoff's separation of thought and action was a part of his conscious desire to fight only one battle, the battle of mathematics.

Birkhoff was uncompromising in his appraisal of mathematicsby the test of originality and relevance. For him the systematic organization or exposition of a mathematical theory was always secondary in importance to its discovery. I recall his remarks on a mathematical treatise that had come to his attention and eventually had a wide circulation but which he did not regard as original. Birkhoff said "I read this book through in a half hour." His choice of topics of investigation could be called objective rather than subjective. His problems were not necessarily chosen from among those which he could solve; indeed many of his papers from 1920 on were on problems which he left unsolved. Some of the current mathematical theories were regarded by Birkhoff as no more than relatively obvious elaborations of concrete examples.

The major interest in Birkhoff in the future will undoubtedly center about his mathematical theories and this report is devoted to their exposition in brief. ${ }^{1}$

Popular opinion focuses attention on two striking achievements, his proof of Poincaré's Last Theorem and the Ergodic Theorem. By contrast much of his other work is obscured. Important as they are, it is to be doubted whether either of the above theorems is more substantial than other phases of his work. His magnificent theory of the generalized Riemann problem, his contributions to difference equations, his formal theory of stability in dynamics, and the con-

\footnotetext{
${ }^{1}$ In reviewing the research of Birkhoff the writer has had the assistance of Dr.
} Pesi Masani, particularly in the study of Birkhoff's early work. 
sequent geometrical theory, all involve structures upon which much can and probably will be built.

In the domain of transitivity in which the Ergodic Theorem lies, the basic hypothesis of metric transitivity remains a conjecture as far as analytic dynamical systems of classical type are concerned. The relative importance in mathematics of statistical or symbolic dynamics can hardly be assessed until it is known whether or not topological transitivity in an analytic system implies metric transitivity. The importance which Birkhoff attached to symbolism in dynamics is made evident by the fact that his last papers on dynamics were largely concerned with the search for a general symbolism characterizing a dynamical system.

Birkhoff's first paper [1 $]^{2}$ was written with H. S. Vandiver under the title $O n$ the integral divisors of $a^{n}-b^{n}$. Birkhoff was eighteen and Vandiver twenty. An immediate consequence of the principal theorem was that there are an infinity of primes congruent to $1, \bmod n$, a result known to Kronecker, Hilbert and Sylvester. The principal theorem is new. One consequence of this collaboration was the high regard for Vandiver's work which Birkhoff held throughout his life.

To continue with a systematic review of Birkhoff's work it will be convenient to divide his papers into six principal groups. Various subjects such as the four-color problem will be referred to separately.

\section{Asymptotic expansions, boundary Value PROBlems, STURM-LIOUVILLE THEOREMS}

(a) Asymptotic expansions and boundary value problems. The years of Birkhoff's graduate study were major years in the development of integral equations. Parallel developments in terms of Green's functions were of great interest. Self-adjoint problems corresponding to symmetric kernels had been extensively studied, but when $n>2$ the case of a non-self-adjoint operator

$$
L(z)=\frac{d^{n} z}{d x^{n}}+*+p_{2}(x) \frac{d^{n-2} z}{d x^{n-2}}+\cdots+p_{n}(x) z \quad(a \leqq x \leqq b)
$$

had not been adequately treated.

The $n$ boundary conditions in [3]

$$
W_{i}(u)=0 \quad(i=1, \cdots, n)
$$

were linear and homogeneous in $u$ and its first $n-1$ derivatives taken at $x=a$ and $x=b$, and were linearly independent. The functions $p_{i}(x)$

2 Numbers in brackets refer to the Bibliography at the end of this paper. 
with all their derivatives were assumed continuous on the closed interval $(a, b)$. The operator $M(z)$ adjoint to $L(z)$ was defined in the classical way. With $L(u)$ and the conditions (1.1) Birkhoff was the first $^{3}$ to associate the adjoint boundary conditions

$$
V_{i}(v)=0 \quad(i=1, \cdots, n)
$$

in the now accepted manner. The parameter $\lambda$ was introduced, giving the "problem"

$$
L(u)+\lambda u=0, \quad W_{i}(u)=0 \quad(i=1, \cdots, n)
$$

and the adjoint problem,

$$
M(v)+\lambda v=0, \quad V_{i}(v)=0 \quad(i=1, \cdots, n) .
$$

In a paper [2] which constituted the first part of his thesis, Birkhoff had given an elegant asymptotic representation of solutions of differential equations of the form

$$
\frac{d^{n} z}{d x^{n}}+\rho a_{n-1}(x, \rho) \frac{d^{n-1} z}{d x^{n-1}}+\cdots+\rho^{n} a_{0}(x, \rho) z=0
$$

for large values of $|\rho|$. The differential equations in (1.2) and (1.3) took the form (1.4) on setting $\lambda=\rho^{n}$. With the aid of these asymptotic representations Birkhoff proved the existence of an infinite sequence $\lambda_{1}, \lambda_{2}, \cdots$ of characteristic values with corresponding solutions

$$
\begin{aligned}
& u_{1}(x), u_{2}(x), \cdots, \\
& v_{1}(x), v_{2}(x), \cdots
\end{aligned}
$$

of (1.2) and (1.3) respectively. He was able to do this by imposing certain determinant conditions on the coefficients in the boundary conditions.

The function $f(x)$ to be expanded was assumed real and piecewise continuous with a continuous derivative. In the case in which the $\lambda_{i}$ are zeros of the first order of the determinant $\Delta(\lambda)$ defining the characteristic roots, the expansion

in which

$$
\sum_{i=1}^{\infty} c_{i} u_{i}(x)
$$

$$
c_{i}=\frac{\int_{a}^{b} f(x) v_{i}(x) d x}{\int_{a}^{b} u_{i}(x) v_{i}(x) d x}
$$

${ }^{3}$ As stated by Bôcher, Applications and generalization of the conception of adjoint systems, Trans. Amer. Math. Soc. vol. 14 (1913) pp. 403-420. 
converges (as does a Fourier expansion) to

$$
\frac{f(x+0)+f(x-0)}{2}
$$

at each inner point of $(a, b)$, to

$$
a_{1} f(a+0)+a_{2} f(b-0)
$$

at $x=a$ and to

$$
b_{1} f(a+0)+b_{2} f(b-0)
$$

at $x=b$ where $a_{1}, a_{2}, b_{1}, b_{2}$ are constants, independent of $f(x)$. In case $\lambda_{i}$ is not a zero of the first order of $\Delta(\lambda)$ the corresponding term in (1.5) is to be replaced by a term readily defined with the aid of the Green's function. The proof of the convergence depended, as with Poincaré, on the method of contour integration in the $\lambda$-plane.

The interest in this work of Birkhoff is signaled by a historic interchange between Birkhoff and J. D. Tamarkin. Tamarkin had independently worked out asymptotic representations similar to those of Birkhoff without publishing them, and was prepared to treat the problem of Birkhoff when he received reprints from Birkhoff. Birkhoff's papers were published in 1907 and 1908 respectively and Tamarkin's paper ${ }^{4}$ was published in 1912. In this paper Tamarkin stated that he found Birkhoff's reasoning brief, and on certain points insufficient. It should be stated that Birkhoff had treated the case of an even $n$ in detail and had indicated the proofs when $n$ was odd. In twelve pages in [6] Birkhoff showed that his earlier proofs could be carried through in general. In a note ${ }^{5}$ in the Rendiconti, Tamarkin reviewed the matter at issue and accepted Birkhoff's supplementary proof.

Problems similar to those treated in [2] and [3] were taken up at length in Birkhoff's and Langer's paper [8] where the case of a system of ordinary differential equations was treated. In this paper may be found references to extensions of the theory by Hopkins and Wilder, pupils of Birkhoff.

(b) Separation and comparison theorems. The existence of characteristic roots in boundary value problems in the plane and the oscillation properties of characteristic functions are treated in [4] follow-

4 J. D. Tamarkin, Sur quelques pointes de la théorie des équations différentielles linéaires ordinaries et sur la généralization de la serie de Fourier, Rend. Circ. Mat. Palermo vol. 24 (1912) pp. 345-382.

${ }^{6} \mathrm{~J}$. D. Tamarkin, Sur un problème de la théorie des équations différentielles linéaires ordinaires, Rend. Circ. Mat. Palermo vol. 37 (1914) pp. 376-378. 
ing the methods of Sturm, Liouville, and Bôcher $(n=1)$. The existence of infinitely many characteristic roots is established in the self-adjoint case and a general oscillation theorem is obtained.

Around 1930 the new techniques auxiliary to the "variational theory in the large" opened up the separation-comparison-oscillation theory for self-adjoint systems of $n$ second-order ordinary linear differential equations. The Sturm separation theorem was generalized by the theorem that the conjugate points on an arbitrary interval $J$ of a point $x=a$ of the $x$-axis differ in number by at most $n$ from the conjugate points on $J$ of a point $x=b$. The boundary conditions were expressed in terms of a minimum set $(u)$ of parameters and an accessory quadratic form $q(u)$ defined in the second variation. A necessary and sufficient condition for the existence of infinitely many characteristic roots was obtained.

In these terms it was possible for the first time to give a systematic classification of the possible separation, comparison and oscillation theorems, and with the aid of Morse's "index form" to establish the principal results. In [9] Birkhoff and Hestenes turned from the older methods of Sturm to the new methods, adding an isoperimetric interpretation to the proofs. A detailed review of [9] is here impossible. Fortunately, a recent "hour address" by W. T. Reid" covers this field in an excellent manner both historically and mathematically, and the interested reader is referred to this address.

In a short note [7] in 1917 Birkhoff gave a simple condition that a normalized set of orthogonal functions $u_{i}(x)$ be closed, and applied this to Sturm-Liouville series. The principle involved in the application could be stated as follows: any set of orthogonal vectors in a functional space lying near enough (in a sense defined by Birkhoff) to a complete set in that space is itself complete. Birkhoff was here sampling a great theory, a theory in which the name of his pupil and colleague Marshall Stone was later to become illustrious.

Perhaps the most remarkable of the contributions of Birkhoff in the domain of separation and comparison theorems is in [5] and concerns an ordinary third order linear differential equation. This equation does not lend itself to treatment by the Sturmian methods nor has it yet been effectively brought into the domain of the variational theory. A solution

$$
y_{i}(x)
$$$$
(i=1,2,3)
$$

is thought of as a curve in a 2 -dimensional projective space. Birkhoff

${ }^{6} \mathrm{~W}$. T. Reid, Boundary value problems of the calculus of variations, Bull. Amer. Math. Soc. vol. 43 (1937) pp. 633-666. 
utilizes the projective differential geometry of Darboux and Wilcynski and by elegant methods far removed from all other methods arrives at theorems very similar to the classical ones. This part of the field deserves further study.

\section{LINEAR DIFFERENTIAL EQUATIONS AND THE GENERALIZED RIEMANN PROBLEM}

With Gauss, Riemann, and Poincare leading the way, ordinary linear differential equations of the second order of Fuchsian type have been studied by almost every analyst of note. In mathematical physics, equations of this type bear the name of Bessel and Legendre. In complex function theory such equations lead by way of conformal mapping to the elliptic modular and automorphic functions. In real function theory integral relations such $\mathrm{as}^{7}$

$$
\int_{0}^{\infty}\left\{4\left(y^{\prime}\right)^{2}-y^{2} / x^{2}\right\} d x>0 \quad(y(0)=0)
$$

are typical of a class of relations which arise ${ }^{8}$ from Fuchsian differential equations with two singular points. Much of Bôcher's research was concerned with these equations. It was natural therefore that Birkhoff should try his hand in this central field.

Departing somewhat from the order in which Birkhoff's papers on this subject appeared we refer first to [11] on $A$ simplified treatment of the regular singular point. At a regular singular point the canonical solutions have singularities (if any) of a well known type. This fact had been previously proved by the method of Frobenius, whereby the coefficients of a formal solution were first obtained and the solution then proved convergent. Birkhoff gives a direct proof, without use of a series expansion, and this proof is barely two pages in length.

Birkhoff was not content with this achievement. Linear differential systems with irregular singular points presented a field of great difficulty and next challenged his attention.

No mathematician of Birkhoff's day possessed greater facilitiy in combining formal algebraic methods with penetrating numerical analysis. Birkhoff needed all of this power in attacking the irregular case. Thomé was his predecessor in using formal solutions of a general type. Poincaré and Horn ${ }^{9}$ had effectively introduced asymp-

${ }^{7}$ Hardy, Littlewood, Polya, Inequalities, p. 175.

${ }^{8}$ Marston Morse and Walter Leighton, Singular quadratic functionals, Trans. Amer. Math. Soc. vol. 40 (1936) pp. 252-286.

${ }^{9}$ See Birkhoff's papers for references. References which may be found in the papers of Birkhoff are generally omitted. 
totic representations. Birkhoff had used formal series and asymptotic representations in the study of boundary value problems. Hilbert and Plemelj, prior to Birkhoff and unknown to Birkhoff, had already solved one of the matrix problems relevant to the theory. Cunningham had generalized Poincaré's use of Laplace transformations in reducing the problem.

There was thus a great technique available to Birkhoff when he began his research. But with all this power no one had carried the theory through to a triumphant end. Birkhoff in $[10,13]$ took up the problem with typical boldness. He added the concepts of canonical systems of differential equations and of equivalence of such systems. He carried through the asymptotic representation of the matrix solution in the general irregular case, enumerated the essential "characteristics" of a singular point of arbitrary rank, defined and solved the "generalized Riemann problem."

Such were Birkhoff's achievements in this field. Although any explicit formulation is complicated, it is needed if the reader is to sense the depth of Birkhoff's mastery and skill.

The differential equations can be represented in the form

$$
\frac{d y_{i}}{d z}=a_{i j}(z) y_{j} \quad(i, j=1, \cdots, n)
$$

using the summation convention for repeated indices. Here $z$ is a complex variable, and $y_{i}(z)$ the unknown $i$ th component of a solution $(y)$. In any local study the singular point can be taken as the point $z=\infty$. The functions $a_{i j}(z)$ are assumed analytic at $z=\infty$ except at most for poles. The maximum order of these poles is denoted by $q$ and $q+1$ is termed the rank of the singularity $z=\infty$. When $q<-1$ the singular point is ordinary. When $q=-1$ the point is regular, and when $q \geqq 0$ irregular. The irregular case $q=0$ had been reduced to the regular case $q=-1$ by Poincaré on using a Laplace transformation of the variables $(y)$, and Poincaré had indicated a possible reduction of the irregular case $q>0$.

To simplify the system (2.1) Birkhoff uses linear transformations of the form

$$
u_{i}=c_{i j}(z) y_{j} \quad(i, j=1, \cdots, n)
$$

in which the functions $c_{i j}(z)$ are analytic at infinity and have there a nonvanishing determinant. Any system into which the system (2.1) goes under (2.2) is said to be equivalent to the system (2.1) at infinity. Birkhoff shows that a system (2.1) of rank $q+1 \geqq 0$ is equivalent to $a$ canonical system 


$$
z y_{i}^{\prime}=q_{i j}(z) y_{j} \quad(i, j=1, \cdots, n)
$$

again of rank $q+1$ in which $q_{i j}(z)$ is a polynomial of degree at most $q+1$.

In proving this theorem Birkhoff used a lemma on analytic matrices [12]. Unknown to Birkhoff at the time, this lemma followed from general theorems of Hilbert and Plemelj. The canonical systems (2.3) are of great value in formally reducing the complexity of the system.

With Thomé, $n$ formal solutions each of the type

$$
y_{i}=e^{p(z)} z^{\mu} B_{i}(z) \quad(i=1, \cdots, n)
$$

are next determined. Here $B_{i}(z)$ is a formal series of nonpositive powers of $z$ such that at least one of the $n$ constant terms $(i=1,2, \cdots, n)$ is not zero while $p(z)$ is of the form

$$
p(z)=\frac{\alpha z^{q+1}}{q+1}+\frac{\beta z^{q}}{q}+\cdots+\lambda z .
$$

The coefficient $\alpha$ is a characteristic root of the matrix of coefficients of $z^{q+1}$ in the polynomials $q_{i}(z)$. These roots $\alpha_{i}$ are assumed distinct. For each of the $n$ formal solutions there is thus a polynomial $p(z)$, an exponent $\mu$, and a formal series $B_{\imath}(z)$. These solutions with $j=1, \cdots, n$ may be chosen so that their determinant $\left|B_{i j}(\infty)\right| \neq 0$.

By making generalized Laplace transformations of the variables $y_{i}$ in (2.3) together with other linear transformations of elementary character, Birkhoff shows that the rank of the differential system at its different singular points can be reduced step by step until the system is regular. This process leads to solutions in the form of generalized Laplace integrals. Each of these integrals is asymptotically represented by a formal solution of the type (2.4) in an appropriate "sector" of a neighborhood of $z=\infty$. These sectors are bounded by the $m=n(n-1)(q+1)$ rays on which $(R=$ real part)

$$
R\left[z^{q+1}\left(\alpha_{i}-\alpha_{j}\right)\right]=0 \quad(i \neq j ; i, j=1, \cdots, n) .
$$

A base $W_{r}$ of these solutions can be chosen in the $r$ th sector $(r=1, \cdots, m)$ so that the base $W_{r}^{*}$ belonging to the succeeding sector is obtained from the base $W_{r}$ by multiplying $W_{r}$ on the right by a matrix of the form $I+C_{r}$, where $I$ is the unit matrix and $C_{r}$ is a matrix with just one non-null element $c_{r}$.

These "transformation constants" $c_{1}, \cdots, c_{m}$, together with the $n$ exponents $\mu_{i}$ and the $n(q+1)$ constants

$$
\left(\alpha_{i}, \beta_{i}, \cdots, \lambda_{i}\right) \quad(i=1, \cdots, n),
$$


of which the $i$ th set appears in the $i$ th solution of a formal matrix solution, are termed by Birkhoff the characteristic constants of the singular point at infinity. With these constants given he shows that there exists a locally defined canonical system (2.3) to which these constants will be equivalent in the above sense.

The preceding theorem completes the local analysis and Birkhoff turns next to the problem in the large. Let $S$ be a differential system of the type (2.1) with at most a finite number of regular or irregular singular points

$$
a_{1}, \cdots, a_{k}
$$

of ranks

$$
q_{1}, \cdots, q_{k}
$$

respectively. Corresponding to a base $Y$ of solutions of $S$ there exist matrices $T_{1}, \cdots, T_{k}$ of constants such that $Y$ is replaced by $Y T_{i}$ when a positive circuit of $a_{i}$ is made. The matrices $T_{i}$ generate the monodromic group $G$ with a relation

$$
T_{1} \cdots T_{k}=I
$$

corresponding to a circuit which encloses the points (2.7).

Birkhoff says that two matrices $Y_{1}(z)$ and $Y_{2}(z)$ whose elements are analytic in the vicinity of $z=a$ are properly equivalent at $z=a$ if

$$
Y_{1}(z)=A(z) Y_{2}(z)
$$

and $A(z)$ is a matrix of elements analytic at $z=a$ with $|A(a)| \neq 0$, and improperly equivalent if (2.10) holds and the elements of $A(z)$ are analytic at $z=a$ except at most for poles. He then proves the following theorem:

Let $a_{1}, \cdots, a_{k}$ be $k$ given points. Let $T_{1}, \cdots, T_{k}$ be matrices of constants such that (2.9) halds. Let $Z_{i}(z)(i=1, \cdots, k)$ be matrices of functions, analytic of determinant not zero in the vicinity of $z=a_{i}$ and undergoing a transformation to $Z_{i}(a) T_{i}$ as $z$ makes a positive circuit of $a_{i}$. There exists a matrix $Y(z)$ of functions not zero for $z \neq a_{1}, \cdots, a_{k}$ and analytic save at these points, which undergoes a transformation to $Y T_{i}$ as $z$ makes a positive circuit of $a_{i}$; furthermore $Y(z)$ is properly equivalent to $Z_{i}$ at $a_{i}(i=1, \cdots, k-1)$ and properly or improperly equivalent to $Z_{k}$ at $a_{k}$.

This theorem contains as a special case a theorem of Hilbert and Plemelj in which the $T_{i}$ 's are given but not the $Z_{i}$ 's, while $Y(z)$ is affirmed to exist and possess elements which are analytic to finite order. The above theorem of Birkhoff gives a first type of generaliza- 
tion of the Riemann problem. A second type of generalization which includes two problems proposed by Riemann is formulated by Birkhoff as follows.

To construct a system of linear differential equations of the first order with prescribed singular points (2.7) and with a given monodromic group, the characteristic constants being assigned for each singular point.

In order that a solution exist it is necessary that the relation (2.9) hold and that the elementary divisors of $T_{i}$ be those of the transformation matrix determined at $a_{i}$ by the "transformation constants" belonging to $a_{i}$. When these conditions are satisfied Birkhoff shows that the problem admits a solution provided one replaces the exponents $\mu_{i}(i=1, \cdots, n)$ belonging to some one of the singular points by new exponents $\mu_{i}+s$ where $s$ is a suitable integer independent of $i$.

This concludes Birkhoff's memorable work on differential systems. A byproduct was a number of theorems on the representation of analytic matrices. Birkhoff returned to this subject in a paper [14] on Infinite products of analytic matrices where he generalized the theorems of Weierstrass and Mittag-Leffler on the representation of an analytic function by an infinite product.

\section{LINEAR DIFFERENCE EQUATIONS}

In his study of the Riemann problem Birkhoff had mastered and extended the use of formal solutions and asymptotic representations. He had used the formal expansions not only to obtain asymptotic solutions but to find the invariant characteristics of the singular points and the a priori limitations on the monodromy group. This use of formal solutions was to remain characteristic of his mode of attack in at least three other main fields, linear difference equations, stability in dynamical systems, and surface transformations with an invariant integral. The underlying analogies were great but the innovations which remained to be made in each case were much greater and would discourage most mathematicians.

In his Fifty years of American mathematics [54] Birkhoff writes that it was the lectures of Van Vleck at Wisconsin on difference equations that led him to an appreciation of the open problems in this field. The thesis of Carmichael in 1911 under Birkhoff at Princeton was perhaps the first significant American contribution on difference equations. Birkhoff's own work began with [15], published in 1911 in a volume of the Transactions that also contained Carmichael's thesis.

Birkhoff [15] dealt ${ }^{10}$ with a system of $n$ linear homogeneous differ-

${ }^{10}$ The writer is indebted to C. R. Adams for a summary of Birkhoff's work on difference equations. 
ence equations of the first order rather than with a single equation of the $n$th order studied by Carmichael. The system was assumed regular in the sense that the characteristic roots were simple and neither 0 nor $\infty$. In matrix form the system took the form

$$
Y(x+1)=A(x) Y(x) \quad(|A(x)| \not \equiv 0)
$$

with $A(x)$ a square matrix of rational functions. Two formal matrix solutions arose

$$
\begin{aligned}
& A^{-1}(x) A^{-1}(x+1) \cdots, \\
& A(x-1) A(x-2) \cdots
\end{aligned}
$$

leading to two "principal solutions" $Y_{+}(x)$ and $Y_{-}(x)$. These principal solutions were analytic except for poles in the finite $x$-plane, and were asymptotically represented in the left and right hand planes respectively by formal solutions. Results similar in character to these had been established by Nörlund and Galbrun a few months earlier using methods based on Laplace transformations.

The matrices of constants which are used in the theory of linear differential systems are here replaced by matrices $P(x)$ of functions with a period 1. In particular a matrix $P(x)$ of this type was determined such that

$$
Y_{-}(x)=Y_{+}(x) P(x) \text {. }
$$

The "characteristic constants" of the system include the constants in $P(x)$ and certain other constants defined by formal solutions in series form. The "generalized Riemann problem" was to determine a system of difference equations whose principal solutions have the given characteristic constants. Properly modified, this problem and its analogues for $q$-difference equations were solved in [13].

The irregular case remained unsolved at this time although Adams had carefully examined the extent to which Birkhoff's methods so far published were sufficient. In [16] (1930) Birkhoff presented a complete solution of the formal problem with strong analogies with the corresponding solution in differential equation theory. His last major paper [17] in this field was a significant collaboration with Trjitzinsky which extended and modified Birkhoff's earlier methods introducing new devices adequate for the analytic treatment of the general irregular case.

Birkhoff's writings in this field occurred in two short periods seventeen years apart. His interest in the field remained keen throughout his life as is shown by the doctoral theses written under him. His students in this field include R. D. Carmichael and K. P. Williams at Princeton; P. M. Batchelder, C. R. Adams, O. E. Lancaster, P. C. 
Guenther and Jeanne S. LeCaine at Harvard. The last two students received their degrees in 1941.

\section{Dynamical systems}

As with Poincare the history of Birkhoff's researches in dynamics is one of successes which are partly complete and partly incomplete. The grand aim was to give a formal normal reduction of a dynamical system which distinguishes equilibrium points as to stability, and enables one to pass from these forms to a complete qualitative characterization of the system. Hamiltonian and Pfaffian systems formed the central core with the restricted problem of three bodies $[20,32$, 39] a typical example. The formal aspects of Birkhoff's research in dynamics may be regarded as relatively complete. Normal forms of great generality were obtained.

On the qualitative side Birkhoff's success was unconditional in many important phases. His central and recurrent motions belong to a theory which is valid for systems with any degree of freedom. His ergodic theorem is, however, conditioned in its usefulness by the uncertainty as to the hypothesis of metric transitivity. By far the greater part of his qualitative analysis was restricted to systems with two degrees of freedom, $m=2$; this is inherent in his dependence on "regular surfaces of section" whose existence seems likely for $m=2$ but is in general unproved, and whose existence for $m>2$ is doubtful. Assuming metric transitivity and the existence of surfaces of section $(m=2)$ he is able to reveal an astonishing range of ordered but intricate dynamical phenomena; in this he is close to realizing a complete, qualitative characterization.

As did Poincaré, Birkhoff seeks to make the periodic orbits central in the theory. Poincaré's conjecture that these orbits are everywhere dense in phase space turns out to be false in special cases but is believed true in general for $m=2$. Poincare was stopped by the inadequacy of his method of analytic continuation of these orbits. In a series of studies Wintner ${ }^{11}$ and others have penetrated deep into the mystery, but in the general case much remains to be done. It is the conjecture of the writer that the method of topological continuation ${ }^{12}$ which Birkhoff does not use may be an essential key to the situation. We shall continue with a summary of various aspects of Birkhoff's theory.

${ }^{11}$ A. Wintner, Grundlagen einer Genealogie der periodischen Bahnen im restringierten Dreikörper Problem, Math. Zeit. vol. 34 (1931) pp. 321-349, 350-402.

${ }^{12}$ See Marston Morse, The calculus of variations in the large, Amer. Math. Soc. Colloquium Publications, vol. 18, New York, 1934, chap.9. 
(a) Formal dynamics. In general form a dynamical system is locally defined by differential equations [26]

$$
\frac{d x_{i}}{d t}=F_{i}\left(x_{1}, \cdots, x_{n}\right) \quad(i=1, \cdots, n) .
$$

The functions $F_{i}$ are assumed analytic in the neighborhood of an initial point $\left(x^{0}\right)$. Hamiltonian systems have the special form

$$
\frac{d p_{i}}{d t}=-\frac{\partial H}{\partial q_{i}}, \quad \frac{d q_{i}}{d t}=\frac{\partial H}{\partial p_{i}} \quad(i=1, \cdots, m)
$$

where $H$ is a function of $2 m$ variables

$$
\left(p_{1}, \cdots, p_{m} ; q_{1}, \cdots, q_{m}\right) .
$$

Pfaffian systems are defined by the Euler equations of an integral

$$
\int_{t_{0}}^{t_{1}}\left[X_{j}\left(x_{1}, \cdots, x_{2 m}\right) x_{j}^{\prime}+Z\left(x_{1}, \cdots, x_{2 m}\right)\right] d t
$$

$i_{n}$ the variational problem in nonparametric form in the space $\left(x_{1}, \cdots, x_{2 m}, t\right)$. In order to be able to solve the Euler equations for the $x_{j}{ }^{\prime}$ it is assumed that the determinant of coefficients of these variables in the Euler equations is not zero. The Hamiltonian and Lagrangian equations also admit the Hamilton and Jacobi variational forms.

Birkhoff speculates on the meaning of these variational forms. His views seem to have evolved somewhat with the passage of time. In [26] he points out that these variational forms have no "special significance" near a point which is not a point of equilibrium. At a point $\left(x^{0}\right)$ of equilibrium

$$
F_{i}\left(x^{0}\right)=0 \quad(i=1, \cdots, n)
$$

in (4.1). He indicates the formal convenience of the variational form when a transformation of coordinates is to be made. He later shows [25] that the Hamiltonian form is one to which (4.1) can be formally reduced neighboring a point of completely formal stability of general type (to be defined later) provided the formal transformations used are sufficiently general in character. Finally in 1943 [44, p. 310] he writes as follows:

"It is only the fact that there is a single explicit variational form available in the entire domain of the independent variables that is really significant. Possibly this interesting situation indicates that the basic importance of variational principles will be found to be topological." (Italics are Birkhoff's.) 
The situation is relatively clear in ordinary dynamics. The writer wonders in what form topology will first appear in quantum mechanics.

Most of Birkhoff's work is concerned with systems (4.1) which involve $t$ in the functions $F_{i}$. This occurs whenever a periodic ${ }^{13}$ orbit $x_{i}=x_{i}(t)$ is given and one then makes a change of coordinates

$$
x_{i}=x_{i}(t)+\bar{x}_{i} \quad(i=1, \cdots, n) .
$$

Starting with (4.1) in its original form this transformation leads to a new function $F_{i}$ of $(\bar{x})$ and of $t$ as well, which vanishes at the origin in the space $(\bar{x})$ for all values of $t$. The origin $(\bar{x})=0$ is then termed a point of generalized equilibrium.

Neighboring such a point the modified system (4.1) is subjected to formal transformations in which $x_{i}$ is replaced by a formal series in new variables $y_{i}$ with coefficients which are analytic and periodic in $t$. These coefficients are permitted to have complex values but in such cases the transformation is to be such that the new variables $y_{i}$ can be arranged in pairs which are formally conjugate in terms of the original real variables. In the case of the Hamiltonian system the new variables $\left(p_{i}, q_{j}\right)$ are taken as conjugate.

Corresponding to an equilibrium point the equation $\mathrm{s}^{14}$ of variations of (4.1) can be brought to a canonical form. In the so-called general ${ }^{15}$ case this canonical form is

$$
\frac{d y_{j}}{d t}=\lambda_{j} y_{j} \quad(j \text { not summed }=1, \cdots, n)
$$

where the $\lambda_{i}$ 's satisfy no relation

$$
m_{1} \lambda_{1}+\cdots+m_{n} \lambda_{n}+m_{n+1}(-1)^{1 / 2}=0
$$

in which the $m_{j}$ 's are integers not all zero. In the case of the Hamiltonian or Pfaffian system the multipliers can be grouped in $m$ pairs

$$
\left(\lambda_{1},-\lambda_{1}\right), \cdots,\left(\lambda_{m},-\lambda_{m}\right)
$$

where the $\lambda_{i}$ 's are either real or pure imaginary. With this understood a prime result of Birkhoff is as follows [26].

By suitable formal (generally divergent) transformations a Hamiltonian or Pfaffian system with a generalized equilibrium point of "general" type at the origin may be taken into a normal Hamiltonian form in which

${ }^{13}$ It will be convenient to take this period as $2 \pi$ throughout.

${ }^{14}$ Involving the terms of first order in (4.1).

${ }^{16}$ The "general" case is referred to throughout in this sense. 
$H(p, q)$ is a formal power series in the products $\left(p_{i} q_{i}\right)$ starting with the quadratic terms

$$
\lambda_{j} p_{j} q_{j} \quad(j=1, \cdots, m) .
$$

The case in which $H$ reduces to the quadratic terms (4.6) is termed degenerate. Birkhoff is largely concerned with a "generalized equilibrium point" of "general" type with a "non-degenerate" Hamiltonian function $H$, as these terms have been defined.

The representations (4.1) which have been described are local. The whole of "phase space" is supposed "covered" by a finite number of such local representations each defined in a region of a space of parameters $(x)$. The phase space $M$ is here supposed to be compact. A transformation $T$ of $M$ is defined in which each point $P$ corresponds under $T$ to the point $P_{t}$ on the trajectory through $P$. The origin for $t$ is so selected that $P_{0}=P$. This one-parameter family of transformations $T$ is called a flow. In the case of certain systems (that is, Hamiltonian systems) there exists an integral of the form $f(V)$ where $V$ is a measurable subset of $M$ such that

$$
f(V)=f(T(V))
$$

where $T(V)$ is the image of $V$ under $T$. The integral $f(V)$ is then termed invariant and the system conservative. The integrand of $f$ is supposed positive and may ordinarily be taken as analytic.

Various types of stability are met, and these must be carefully distinguished. Historically, one of the most important is the property of a conservative system whereby the images of any open region $R$ under the flow intersect $R$ for values of $t$ which cluster at $\infty$.

First order formal stability is defined relative to a generalized equilibrium point of a Hamiltonian or Pfaffian system. It requires that the multpliers $\lambda_{i}$ be purely imaginary.

Formal trigonometric or complete stability occurs as follows. Let $T$ be a time interval, $m$ any positive integer, $P_{s}$ any polynomial in $(x)$ with coefficients analytic and periodic in $t$ and of lowest degree $s$. It is then possible, for $e>0$, to approximate $P_{s}$, for time intervals less than $T$, with an error numerically less than $M e^{m+s}$ with a suitably chosen sum,

$$
\sum_{j=0}^{N}\left(A_{j} \cos k_{j} t+B_{j} \sin k_{j} t\right) \quad\left(k_{i}-k_{j}>k>0\right)
$$

in which $M, N, k$ are constants dependent only on $m$ and $P_{s}\left(k_{0}=0\right)$.

(A) It is a capital result of Birkhoff that first order formal stability of Hamiltonian or Pfaffian systems in the case of a point of "generalized 
equilibrium" of "general" type implies formal trigonometric stability [26].

(b) Recurrence and transitivity. A subset $S$ of the phase space $M$ which is carried into itself by the "flow" on $M$ is called invariant. A point $P$ of $S$ is called wandering relative to $S$ if there exists a neighborhood $W$ of $P$ relative to $S$ which, for some $t_{0}>0$, and for $t>t_{0}$, has no image which intersects $W$. A maximal invariant subset $S^{*}$ of $S$ whose points are non-wandering relative to $S^{*}$ is called central in $S$. Birkhoff shows by transfinite induction that every non-empty invariant subset $S$ of $M$ possesses a central subset $S^{*}$. Such a set $S^{*}$ is closed relative to $S$ and non-empty. If the system is conservative as defined earlier, the set which is central relative to $M$ is $M$; but if $M$ is nonconservative, the central set $M^{*}$ of $M$ is in general a proper subset of $M$. Let $N$ be an arbitrary neighborhood of $M^{*}$ on $M$. The fraction of time which the finite $\operatorname{arc} P_{0} P_{t}$ spends in $N$ tends to 1 as $t$ becomes infinite, independently of the choice of $P_{0}$ on $M$ [26].

A closed invariant subset of $M$ no proper subset of which is a closed invariant set is called minimal and any sub-motion (complete trajectory) of the set is called recurrent [18]. A periodic motion is a very special minimal set. For a motion $P_{t}$ to be recurrent it is necessary and sufficient that for every $e>0$ there exists a $T>0$ so large that any arc $P_{t} P_{t+T}$ of the motion has points within a distance $e$ of every point of the motion. Limit points of a motion $P_{t}$ as $t$ becomes negatively or positively infinite respectively are called $\alpha$ - and $\omega$-limit points respectively. Birkhoff shows that the $\alpha$ - (or $\omega$-) limit motions of a given motion include at least one minimal set. In general, minimal sets do not reduce to a periodic motion. Recurrent motions on surfaces of negative curvature can be studied and classified with the aid of the topological symbolism of Hadamard, ${ }^{16}$ as Morse first showed in his thesis.

In his desire to determine the ultimate significance of formal stability of Hamiltonian or variational forms, Birkhoff goes beyond the case of Hamiltonian or Pfaffian systems. In such general dynamical systems (A) is not true. Birkhoff defines even more general formal transformations and states that if these be admitted, any dynamical system can be formally reduced near an equilibrium point of general type to Hamiltonian form provided the conditions of first order and formal trigonometric stability are satisfied [25]. The meaning of this bold step is not altogether clear.

Departing from the domain of formal operations, permanent stabil-

${ }^{16}$ For a general theory see Marston Morse and Gustav A. Hedlund, Symbolic dynamics, I and II, Amer. J. Math. vol. 60 (1938) pp. 815-866; vol. 62 (1940) pp. 1-42. 
ity is said to occur when sufficiently small initial displacements at $t=t_{0}$ from an initial solution cause displacements from the initial solutions which are arbitrarily small for all time. Formal conditions for permanent stability are not known. In particular it is not known whether or not a point of generalized equilibrium which is formally "completely" stable is in general permanently stable. The theory in the large is tied up with the theory in the small because the validity of the hypothesis of metric transitivity in a system would imply that permanently stable periodic orbits do not exist.

A motion is termed transitive if it is everywhere dense in the phase space $M$. If such a motion exists the system is called topologically transitive. Birkhoff exhibits examples of such transitivity. The most general case of topological transitivity established up to this time in dynamical systems of classical type is that of geodesic motion on a surface of genus greater than 1 on which no point has a conjugate point. ${ }^{17}$

Under metric transitivity, as first defined by Birkhoff and P. A. Smith [27], the only invariant subsets of $M$ are sets whose measure is 0 or $m(M)$. Metric transitivity implies topological transitivity. The converse is probably true in analytic systems or systems with some degree of analytic regularity. A proof or disproof of this converse is much needed.

Smith and Birkhoff [27] have defined central motions of iterates of a surface homeomorphism and characterized them in various ways. Metric transitivity is shown by them to be equivalent to the condition that no two "invariant integrals" on $S$ be linearly independent. In the general analytic case at least two central motions are shown to exist.

B. O. Koopman [30] discovered that a conservative flow $T_{t}$ on $M$ with an invariant measure on $M$ could be represented by a oneparameter family of unitary transformations in Hilbert space, thus opening up a new mode of investigation of dynamical systems. Following this, von Neumann established his "mean ergodic theorem" appropriate to Hilbert space. Under the stimulus of these ideas, Birkhoff [29] saw that in treating transitivity open sets should be replaced by sets of positive measure. Birkhoff's first theorem is what

${ }^{17}$ Marston Morse and Gustav A. Hedlund, Manifolds without conjugate points. Trans. Amer. Math. Soc. vol. 51 (1942) pp. 362-386. For other aspects of the problem, see J. C. Oxtoby and S. M. Ulam, Measure preserving homeomorphisms and metrical transitivity, Ann. of Math. vol. 42 (1941) pp. 874-920, and P. R. Halmos, In general a measure preserving transformation is mixing, ibid. vol. 45 (1944) pp. 786-792. 
is commonly known as his ergodic theorem. We shall give a formulation due to Khintchine. ${ }^{18}$

The space $M$ is assumed to have a finite measure $m$ invariant under the flow. Let $f$ be integrable over $M$ and let $P$ be a point of $M$. Then

$$
\lim _{T \rightarrow \infty} \frac{\int_{0}^{T} f\left(P_{t}\right) d t}{T}
$$

exists for almost all $P$ on $M$.

Extensions and applications of the ergodic theory have been made by Wiener, Wintner, E. Hopf, Garrett Birkhoff and others. If $f$ is the characteristic function of an arbitrary measurable subset $V$ of $M$, the integral in (4.7) is the measure of the time spent by $P_{t}$ in $V$ for $0 \leqq t \leqq T$, and, in case the system is metrically transitive, Birkhoff shows that the limit in (4.7) equals the ratio of $m(V)$ to $m(M)$ for almost all $P$ on $M$.

(c) Periodic orbits. In the case of a Hamiltonian or Pfaffian system with two degrees of freedom and with a periodic orbit $\lambda$ (period $2 \pi$ ) one can use an integral to reduce the dimension of the phase space containing $\lambda$ to $n=3$. Let $S$ be an element of a regular analytic "surface of section" which cuts across $\lambda$ at some point $Q$. If $P$ is a point of $S$ sufficiently near $Q$ the orbit $P_{t}$ will cut $S$ again later at a point $T(P)$. Poincaré introduced these transformations $T$ of $S$ into itself in order to establish the existence of periodic orbits in the neighborhood of $\lambda$. It is clear that a necessary and sufficient condition that $P$ represent an orbit with a period near $2 \pi$ is that

$$
P=T(P) .
$$

One can similarly use the $k$ th iterate $T^{k}(P)$ of the transformation $T(P)$ to find orbits near $\lambda$ with periods near $2 k \pi$.

The orbit $\lambda$ is termed simple if its equations of variation have no nonzero periodic solution. If $\lambda$ is simple it cannot be a member of an analytic family of periodic orbits, and, bearing in mind no doubt that this excludes systems classically called "integrable," Birkhoff terms the problem non-integrable if every periodic orbit and its multiples are simple [26].

A generalized equilibrium point has been termed degenerate if the Hamiltonian function $H$ can be formally reduced to quadratic terms. A system in which each periodic orbit is nondegenerate is termed nondegenerate. Non-integrable nondegenerate systems exist as Birkhoff shows. Let $(u, v)$ be regular coordinates on $S$ neighboring a fixed

${ }^{18}$ A. Khintchine, $Z u$ Birkhof's Lösung des Ergodenproblems, Math. Ann. vol. 107 (1933) pp. 485-488. 
point $(0,0)$ of $T$. If the system is non-integrable and nondegenerate the terms of first order in $T$ reduce either to a rotation about $(0,0)$ through an angle incommensurable with $2 \pi$ (fixed point of elliptic type) or to the form

$$
u^{\prime}=\rho u, \quad v^{\prime}=\frac{1}{\rho} v \quad(\rho \neq 0 \text { or } 1)
$$

where $\rho$ is real (fixed point of hyperbolic type) [23].

Following Poincaré, Birkhoff makes use of the existence of an invariant integral in the original phase space to obtain an invariant integral $[23,33]$

$$
\iint Q(u, v) d u d v
$$

on $S$ neighboring the origin, where $Q$ is real and analytic in $(u, v)$. Birkhoff shows that there exists a real power series $F(u, v)$ which may be convergent or divergent, and is formally invariant under $T$. The series $F$ starts with terms of the second degree or higher. The series $Q$ and $F$ are formally related by differential equations

$$
Q \frac{d u}{d k}=F_{u}, \quad Q \frac{d v}{d k}=-F_{v}
$$

whose formal solutions $u$ and $v$ starting with terms of the first order yield $T^{k}$ when $k$ is replaced by an integer.

Formal series transformations of $u$ and $v$ reduce $T$ to a transformation

$$
u_{1}+i v_{1}=(u+i v) e^{h(r) i} \quad\left(h(r)=a+c r^{2 m}\right)
$$

when $\lambda$ is simple and nondegenerate and the fixed point $(0,0)$ elliptic. Here $a$ and $c$ are real constants with $c \neq 0$ and $a$ incommensurable with $2 \pi$. The exponent $m$ is a positive integer in general 1 . Whenever (4.8) holds, $T$ is actually as well as formally very close to a rotation through an angle $a$, for sufficiently small positive $r$. For a simple nondegenerate orbit and a hyperbolic fixed point, $T$ has the normal form

$$
u_{1}=\rho u e^{c(u v)^{m}}, \quad v_{1}=\frac{1}{\rho} v e^{-c(u v)^{m}} \quad(m>0 ; c \neq 0)
$$

where $\rho$ is real and neither 0 nor 1 . The use of these forms to obtain approximate representations of $T$ is reminiscent of Birkhoff's use of asymptotic representations in his thesis, in the Riemann problem and in the formal Hamiltonian theory. 
In [33] Birkhoff largely removes the condition that the orbit be "simple" although this task is one of great formal complexity. When the periodic orbit is "multiple" the terms of first order in the representation of $T$ have equal characteristic roots. In such special cases the corresponding fixed point $Q$ of $T$ is termed hyperbolic if there exists a real invariant curve passing through $Q$. Otherwise $Q$ is termed elliptic.

To establish the existence of periodic orbits in the restricted problem of three bodies Poincaré introduced his celebrated "last geometric theorem" but was unable to prove it. ${ }^{19}$ This theorem may be stated as follows:

Given a ring $0<a \leqq r \leqq b$ in the $r, \theta$, plane and $a$ 1-1 continuous areapreserving transformation $T$ of the ring under which points on $r=a$ advance, and those on $r=b$ regress, there will exist at least two points of the ring invariant under $T$.

A number of mathematicians had attempted to prove this theorem and at least one erroneous proof was published. Urged on by his colleagues, Birkhoff [19] gave a beautiful proof of the theorem that went directly to the heart of the problem. Modified forms of the theorem were also presented by Birkhoff [24].

Using theorems of a nature similar to that of Poincaré, Birkhoff proves the following in [26]:

$(\alpha)$ In non-integrable, nondegenerate Hamiltonian systems with two degrees of freedom any neighborhood of a periodic orbit of elliptic type includes infinitely many periodic orbits both of the elliptic and hyperbolic types. At most a finite set of these orbits have periods less than a given constant.

A generalization of the Poincare theorem to higher dimensions is given in Birkhoff [28]. This generalization employs the theorem of Morse that the number of critical points, suitably counted, of an analytic function defined on the product of $n$-circles is $2^{n}$. With D. C. Lewis, Birkhoff's theorem is applied in [31] to establish the existence of periodic orbits neighboring a periodic orbit of general, formally stable type.

A periodic orbit $\lambda$ of hyperbolic type cannot be permanently stable. When $\lambda$ in $\alpha$ is hyperbolic there exist [23] two analytic curves on $S$ passing through $(0,0)$ and invariant under $T(P)$. These invariant curves on $S$ imply the existence of analytic families of motions asymptotic to $\lambda$ in either sense.

No example is known in which the elliptic orbit $\lambda$ of $(\alpha)$ is "perma-

${ }^{19}$ H. Poincare, Rend. Circ. Mat. Palermo vol. 33 (1912) pp. 375-407. 
nently stable" in the geometric sense and none such could exist if the system is metrically transitive. Nevertheless Birkhoff gives a relatively complete description of motions neighboring $\lambda$ in case $\lambda$ is in fact permanently stable. Since this description is similar to the one given by Poincaré, it is omitted.

A priori existence theorems for periodic orbits are very necessary. Birkhoff turns to the "direct" method in the calculus of variations, and uses the Jacobi "action integral" $J$. In Lagrangian problems in which the constraints are independent of the time this integral will in general be regular and positive definite in the sense of classical variational theory. A periodic minimizing extremal will then exist in an "extremal convex" region. No periodic minimizing extremal exists in very general problems, as for example in the restricted problem of three bodies with a parameter $\mu$ sufficiently small. To fill in this gap in the theory Birkhoff applied a "minimax principle" [21].

In Birkhoff's applications this principle reduces to an existence theorem for critical points of an analytic function $F(x)$ of $n$-variables. If one supposes for the sake of definiteness that $F$ is defined over a regular, compact, analytic manifold, then, suitably counted, there exist at least $R_{1}+M_{0}-1$ generalized saddle points, where $R_{1}$ is the linear connectivity of the manifold and $M_{0}$ the number of points (supposed isolated) of relative minimum of $F$. In similar or related forms this principle was known and applied by Poincaré, Maxwell, and Kronecker, and has an origin even more remote in the past. Birkhoff's bold step was to conceive of its application to functions of curves such as the integral $J$. He applied it in the billard ball problem [26] (motion on a convex table) and to obtain closed geodesics on a convex surface. On slipping an elastic band over the surface from one egg-shaped end to the other the process that requires the least stretching leads to a closed geodesic of minimax type. However, Birkhoff's conjecture that the closed geodesic analogously obtained for a topological $n$-sphere is of "minimax type," as characterized by him, is not true if $n>2$.

The a priori existence of periodic orbits is necessary for the dynamical theory as conceived by Poincaré and Birkhoff.

(d) General qualitative dynamical theory. This theory is limited almost wholly to the case of two degrees of freedom $m=2$ largely because a fundamental tool with Birkhoff as with Poincaré is a "regular surface of section." One presupposes a three-dimensional, analytic, compact, nonsingular phase space. A regular surface of section $S$ is then an analytic surface crossed in the same sense by all trajectories without being tangent to $S$ except along the boundaries. These bound- 
aries are to be finite in number and consist of closed trajectories. Further every trajectory except the boundary orbits shall cut $S$ at least once in any sufficiently large fixed interval of time.

It seems likely that such surfaces of section exist in general when $m=2$. As Poincaré indicated, such a surface exists in the restricted problem of three bodies when the mass $\mu$ (that is, earth) is sufficiently small. Although open hyper-surfaces of section exist when $m>2$ it has not yet been found possible to make profitable use of them.

As in (c) the "flow" in the phase space defines and is characterized by a conservative transformation $T$ of $S$ into itself. Birkhoff makes three tentative hypotheses none of which has been proved to hold in general, but each of which probably does so hold. These hypotheses as given in [33] are:

(A.1) The dynamical system is transitive.

(A.2) A regular surface of section $S$ exists.

(A.3) There is at least one point which is fixed under $T$, but any such point, if elliptic, corresponds to a nondegenerate, periodic orbit.

This qualitative theory culminates in the paper [33] 130 pages long, crowned by the Pontifical Academy. This paper resumes and extends much of Birkhoff's earlier dynamical theories. Upon finishing this work Birkhoff commented to the author that it was an exhausting task. In this connection it is of historical interest to recall that Birkhoff stated about 1925 that he considered his Transactions paper [21] for which he received the Bôcher prize as good a piece of research as he would be likely to do. In the writer's opinion, the Rendiconti memoir [20], also a prize paper, should be placed near the top of Birkhoff's works, not because it is final but because one finds Birkhoff there first meeting the problems which he inherited from Poincaré, and taking the first clear cut concrete steps which he later generalized.

The Pontifical memoir can only be broadly summarized. After extensive formal studies already reported on, Birkhoff shows that under hypotheses (A) there exists an infinite number of hyperbolic points fixed relative to some of the iterates $T^{k}$ of $T$. There may exist no elliptic points, but if one exists, there exist infinitely many such elliptic points. The general existence of the hyperbolic fixed points makes them a suitable instrument on which to base a qualitative characterization of $T$ and this Birkhoff proceeds to do.

On $S$ the invariant curves $C$ through a hyperbolic fixed point $P$ are divided into $\alpha$-branches $E_{\alpha}(P)$ and $\omega$-branches $E_{\omega}(P)$ according as the iteration of $T^{-1}$ or $T$ makes a point on $C$ tend toward $P$ on $C$ as a limit. In the original phase space each point on an $\alpha$-branch ( $\omega$-branch) defines a motion which becomes negatively (positively) 
asymptotic to the periodic orbit determined by $P$. Returning to $S$, Birkhoff shows that under hypotheses (A) these $\alpha$ - and $\omega$-branches associated with a single hyperbolic fixed point $P$, if continued analytically, are everywhere dense on $S$. There is an exception which does not occur for most fixed points $P$ when one of these branches belongs to a second hyperbolic fixed point. These $\alpha$ - and $\omega$-branches intersect in infinitely many points termed "homoclinic" by Poincaré. Homoclinic points are proved to be cluster points of fixed points.

In the case of a metrically transitive system the set of all fixed points and of all $\alpha$-or $\omega$-branches attached to hyperbolic fixed points has a measure zero on the surface of section. The remaining points are grouped into sets $\Sigma_{Q}$ termed isomorphic by Birkhoff. Let $Q$ be a point which belongs to no set $E_{\alpha}(P)$ or $E_{\omega}(P)$. The maximal connected set of points which contains $Q$ but no point of a set $E_{\alpha}(P)$ or $E_{\omega}(P)$ is independent of $P$ among hyperbolic fixed points $P$ and is denoted by $\Sigma_{Q}$. These sets are transformed into each other under $T$, hence the term isomorphic. It is probable that $\Sigma_{Q}=Q$ in general; this case is called regular.

Birkhoff shows in what sense the intersections of an $E_{\alpha}(P)$ and an $E_{\omega}(P)$ "characterize" the isomorphic sets $\Sigma_{Q}$. He invents a 2-dimensional symbol called the signature of the system which displays the topology of the intersections of $E_{\alpha}(P)$ and $E_{\omega}(P)$. For Birkhoff such a signature is the ultimate in the qualitative description of a dynamical system.

These $\alpha$-and $\omega$-branches belonging to a hyperbolic point $P$ are properly called asymptotic to $P$. Birkhoff also defines connected sets asymptotic to elliptic points. These are sets and not curves, at least a priori. They were studied at length by Birkhoff before he recognized the importance of the $\alpha$ - and $\omega$-branches belonging to hyperbolic points. He indicates the formal analogies between the sets asymptotic to elliptic points and the $\alpha$ - and $\omega$-branches attached to hyperbolic points.

(e) The restricted problem of three bodies. This famous problem was studied by Birkhoff in three principal papers [20,32, 34]. Much of the work in these papers is an application or exposition of general theories elsewhere reported. There remain several special achievements to be noted.

The two finite bodies (that is, sun and earth) have masses $1-\mu$ and $\mu$ respectively, and the third body $(x, y)$ has a mass which is infinitesimal. The two principal bodies are originally supposed to rotate about their common center of gravity in the clockwise sense. In the representation of the problem in the $(x, y)$-plane the coordi- 
nates have been so transformed that the earth and sun rest fixed at points on the $x$-axis with their center of gravity at the origin. The problem then has the Jacobi integral

$$
\left(\frac{d x}{d t}\right)^{2}+\left(\frac{d y}{d t}\right)^{2}=2 \Omega(x y)-C
$$

where $\Omega$ is a positive function of special nature.

When $C$ is sufficiently large the point $(x, y)$ is confined in "Case I" to an oval about one of the finite masses. The phase space is then shown by Birkhoff to be the homeomorph of the three-dimensional projective plane. Topological models are given for the phase space for other values of $C$. Birkhoff keeps to Case I in general.

Direct periodic orbits had been established by Poincaré only for sufficiently small values of $\mu$. Birkhoff shows that in Case I at least one retrograde periodic orbit symmetric with respect to the $x$-axis always exists. He replaces the ring transformation of Poincare which led to the "last geometric theorem" by a transformation of a diskoid into itself. As long as this construction is possible there exists a direct periodic orbit. But such a construction is definitely established only for sufficiently small values of $\mu$. However the existence of the diskoid seems to demand less by way of proof than the existence of the Poincaré ring.

Returning to the ring transformation of Poincaré, Birkhoff concerns himself with the existence of orbits which are symmetric with respect to the $x$-axis. He shows that the transformation of the ring surface of section $S$ into itself is the product of two involutory transformations. He attaches two characteristic integers to a symmetric periodic orbit as follows. Suppose that this orbit cuts $S$ at a point $P$. If $T$ represents the ring transformation of Poincare there is a least integer $k$ such that the $T^{k}(P)=P$. The $k$-iterates of $P$ on $S$ will rotate about the Poincaré ring $l$ times. The pair $(k, l)$ characterize the orbit. Birkhoff shows that there are infinitely many pairs $(k, l)$ which represent symmetric periodic orbits and he exploits this symbolism to the full.

After reading the preceding report on Birkhoff's advances in dynamics the reader may find it of interest to compare a summary [22] in 1920 and a prospectus [35] in 1941 by Birkhoff himself. The summary occurs under the title Recent advances in dynamics and the prospectus is entitled Some unsolved problems of theoretical dynamics and was presented at a fiftieth anniversary symposium at the University of Chicago in 1941. After a lapse of twenty-one years the problems remain essentially the same, but the approach and particularly 
the language of approach has become more topological and abstract. "Conservative flows" are to be studied both in the topological and the statistical sense, and abstract variational theory is to enter. There is no doubt about the challenge of the field, and the need for a powerful and varied attack.

\section{Physical theories ${ }^{20}$}

The earliest evidence of Birkhoff's interest in relativity can be found in a review of Books on relativity [36], in 1922. In a few words, while commenting upon the development of Einstein's general theory, he formulates what has turned out to be his own ideal in the creation of physical theories: "In the first place, it illustrates afresh the importance of taking the simplest possible case as an abstract basis of departure. Secondly, Einstein uses mathematical analogy in passing, step by step, from the simple universe of the special theory to the most general universe, and at each step the mere sense of mathematical form is sufficient to point the way to a natural generalization. The mathematician may feel satisfied that the formal analogies supplied by classical dynamics and four-dimensional geometry furnish the very basis by which Einstein's generalization proceeds" (page 217).

In 1923, in his widely acclaimed monograph, Relativity and modern physics (written with the cooperation of R. E. Langer), Birkhoff carries out this ideal as closely as he can by establishing the electromagnetic equations, and the fundamentals of the special and general theories of relativity, showing how this can be done without indiscriminate appeal to physical intuition, and with a maximum of regard for geometrical symmetry, mathematical rigor and simplicity.

Feeling that "without a true model as a starting point, it does not seem likely that a final conception of the physical universe can be arrived at" [37, p. 70], Birkhoff selects a "perfect fluid," which satisfies the classical equations of hydrodynamics, slightly modified and unified so as to be invariant under the Lorentz transformations. The pressure-density relation was such that all disturbances would be propagated with the speed of light. This fundamental property of the substance of which all matter is composed was intended to eliminate those paradoxes in the theory of collision which Birkhoff pointed out in his presidential address of 1926 [40].

In this address, Birkhoff urged theoretical physicists to attempt to

${ }^{20}$ This account of Birkhoff's physical theories has been written by Morse's assistant Dr. Richard Arens, who had the advantage of numerous conversations on this subject with Birkhoff in the last years. 
account for atomic phenomena using mainly the ideas of special relativity and abstract dynamics, for he felt that the recurrence properties of dynamical systems could eventually provide an intuitive explanation of quantum effects (cf. also [42, p. 317]). He made a modest attempt in this direction himself $[39,40]$. He welcomed the return to the use of differential equations, due to Schrödinger, and justified these equations, from a general point of view, in [41].

Although it soon appeared that the "perfect fluid" could not be limited to a desirable behavior by its hydrodynamical equations alone [42, p. 324], Birkhoff retains it as raw material for "mathematical model-making," perhaps because he feels that any physical theory should contain some statement about the constitutive equations of "matter" [44, p. 301]. The best cross section of Birkhoff's ideas after ten years of contemplation can be obtained from his Franklin Institute Lecture of 1938, Electricity as a fluid [43], in which he states his ideas, opinions, and preferences with unusual directness, clarity and humor.

The final phase, which was undoubtedly intended to be only the beginning, of Birkhoff's work in relativity was his gravitational theory [43] of 1943. With such a degree of analogy to electrodynamics as the essence of things permits, this theory involves a gravitational tensor potential governed by a linear differential equation which is, as seems proper in view of the underlying flat but Minkowskian space-time, of hyperbolic type. Whereas in electrodynamics the acceleration of a charged particle is a linear function of the velocity with coefficients characterizing the field, here the acceleration depends quadratically on the velocity. The theory is completely linear, and homogeneous except for the presence of the "perfect fluid."

As regards experimental verification, Birkhoff's theory predicts the same rate of rotation of the apse line of planetary orbits as does the general theory of relativity. Recently, moreover, a practical method of successive approximation for treating the two body problem has been worked out. ${ }^{21}$

In Birkhoff's theory, phenomena such as the bending of light around the sun cannot be regarded as of electromagnetic nature, since there is no interaction of electromagnetic and gravitational field; but Birkhoff finds that by considering the world lines of "photons" as limits of world lines of particles on which the speed approaches that of light, the proper magnitudes for the bending of light and red-shift effect can be obtained [44a]. He has also discovered

${ }^{21}$ C. Graef Fernandez, El movimiento de los dos cuerpos en la teoria de la gravitación de Birkhoff, Boletin de la Sociedad Matemática Mexicana vol. 1 (1944) p. 25. 
another approach to the red-shift effect which makes use of the wavelength-energy relation of Planck. These ideas merit careful investigation, even apart from the remaining theory.

It has been stated ${ }^{22}$ that this theory does not provide for the identity of gravitational and inertial mass in as effortless a manner as the theory of Einstein, and this seems to be a defect from which linear theories must suffer. ${ }^{23}$

Birkhoff inherited from Poincaré the sentiment that no single mathematical theory of any phenomenon deserves the exclusive attention of physicists, or at least of mathematicians.

\section{Miscellaneous works}

(a) Fixed points in function space. The paper [45] written jointly with O. D. Kellogg is undoubtedly one of Birkhoff's most important contributions. The first theorems are generalizations of the Brouwer theorem that a continuous transformation of an $n$-disc into itself has at least one fixed point. This is applied to a transformation of a space of uniformly bounded and equicontinuous functions $f(s)$ and of the space of functions $f(s)$ which are summable square and representable by a subset of points in Hilbert space for which $\sum x_{i}{ }^{2}$ converges uniformly. Fixed elements $f(s)$ are inferred.

A second point of departure is the theorem that a continuous transformation of a real projective space of even dimensions into itself has at least one fixed point or "invariant direction." This theorem extends to integral equations.

Deformations of transformations are extensively used. A continuous transformation $T_{\lambda}$ on an $n$-sphere $H_{n}$ into the embedding $(n+1)$ space is given as depending linearly on a parameter $\lambda$ in such a manner that as $\lambda$ varies to $0, T_{\lambda}$ deforms into the identity without intersecting the origin. It is concluded that to each point (b) on $H_{n}$ corresponds a point (a) ("inverse direction") on $H_{n}$ whose image under $T_{\lambda}$ is on the same ray as (b). This theorem has applications in the Fredholm theory.

It is of interest that the paper in the Transactions preceding [45] was an elegant treatment by Alexander of "transformations with invariant points," including many of the theorems in [45] concerning transformations of finite-dimensional spaces but not including applications to function spaces. The work of Birkhoff and Kellogg was the

${ }^{22} \mathrm{H}$. Weyl, Comparison of a degenerate form of Einstein's with Birkhoff's theory of gravitation, Proc. Nat. Acad. Sci. U.S.A. vol. 30 (1944) pp. 205-210, especially p. 205.

${ }^{23}$ Loc. cit. p. 206. 
acknowledged stimulus of the noteworthy extensions of Schauder, and later of Schauder and Leray, including important applications to partial differential equations. The invariance of the Brouwer degree of a transformation under continuous deformations is a unifying element in the Schauder-Leray theory.

It is of interest to recall that Birkhoff's introduction to fixed point theory was by way of the lemma of Poincare that the sum of the signed indices of an analytic vector field on an orientable surface of genus $p$ is $2-2 p$. Birkhoff used this theorem in his theory of elliptic and hyperbolic fixed points of the surface transformations arising in dynamics [21].

(b) The 4-color problem. ${ }^{24}$ Birkhoff's interest in this problem was aroused in Veblen's seminar in "analysis situs" during his years at Princeton. Birkhoff brought out two papers $[46,47]$ on this subject during his stay at Princeton.

In [47] Birkhoff revived the qualitative approach based on Kempe chains. He found certain "reducible configurations," that is, figures whose presence in a regular map reduces the coloration problem to that of a simpler map. Of these the most important and the hardest to analyze was a ring of two regions not surrounding a regular pentagon. Of such reducible configurations later found by Franklin, Errera, and Winn, many are direct extensions of those of Birkhoff and most require for their proof Birkhoff's theorem on rings of five regions. The minimum number of regions in a map not capable of coloration in four colors was shown successively to be at least 25 (1922, Franklin), 27 (1927, Reynolds), 31 (1938, Franklin), and finally 35 (1940, Winn).

In [46] Birkhoff introduced the quantitative approach. "Chromatic polynomials" $P(x)$ equal to the number of ways a given map can be colored in $x$ colors were found. Although the main objective of showing that $P(4)>0$ was not achieved, many properties of $P(x)$ were obtained by Birkhoff $[46,48]$ and later by Whitney whose thesis was on this problem. Birkhoff hoped that the theory of chromatic polynomials could be so developed that methods of analytic function theory could be applied. Birkhoff took the first real step in this direction in [49].

The quantitative approach was not as successful in the study of the classical problem as the qualitative. Nevertheless Birkhoff felt that it might be easier to establish a stronger result concerning $P(x)$ than a direct and weaker theorem of qualitative type. In a long joint paper with D. C. Lewis, submitted to the Transactions, a fusing of the quali-

${ }^{24}$ The writer is indebted to Philip Franklin and to D. C. Lewis for summaries of work on the 4-color problem. 
tative and quantitative approaches is sought by way of a study of $P(x)$, borrowing and modifying the method of Kempe chains. In this study the reducibility of certain configurations previously established by Kempe chains is established by quantitative methods. Deeper insight is thus gained.

This paper includes a conjecture to which Birkhoff attached the maximum importance. The map of the sphere under consideration consists of $n+3$ simply-connected regions containing only triple vertices, that is, points on the boundaries of just three regions. Let $P_{n+3}(x)$ be the number of ways of coloring this map in $x$ colors. It is known that this polynomial is of degree $n+3$ and vanishes when $x=0$, 1 and 2 . Hence

$$
Q(x)=\frac{P_{n+3}(x)}{x(y-1)(y-2)}
$$

is a polynomial of degree $n$ in $x$. The conjecture is that

$$
(x-3)^{n} \leqq Q(x) \leqq(x-2)^{n}
$$

for $x \geqq 4$ and $n>0$. The conjecture is readily established for $x \geqq 5$. It is also established for $n<9$ even when $x=4$. It has been verified for special maps of $12,14,15,16$, and 17 regions with $x=4$.

This paper also contains an asymptotic formula for $P_{n}(x)$ conjectured by Birkhoff as a result of heuristic probability considerations.

(c) General mean value and remainder theorems. The paper [50] of Birkhoff written in 1904 is somewhat complicated in form as is perhaps necessary. There is given the value of $f(x)$ and some of its derivatives up to the $(n-1)$ st at $n$ points on an interval $(a, b)$. Birkhoff introduces a polynomial $F$ which together with its derivatives equals $f$ and its derivatives at the respective points in question. The function $f^{(n-1)}(x)$ is assumed continuous, and $f^{(n)}$ is assumed to exist on $(a, b)$. The general remainder theorem involves $f$ and $F$ and includes most known remainder theorems. It has applications in the fields of mechanical differentiation and quadratures.

(d) Aesthetic measure. Birkhoff's interest in the analysis of art and music forms was of long standing. It culminated in his famous treatise on aesthetic measure [51]. To oversimplify this work of Birkhoff would result in a misrepresentation. For this reason and because of the readability of [51] no extended account of Birkhoff's work in this field will be presented.

Within each class of aesthetic objects Birkhoff defines the order $O$ and complexity $C$ so that their ratio

$$
M=\frac{O}{C}
$$


yields the "aesthetic measure" of any object of the class. Equal at least in importance to the particular value of $M$ attached to an aesthetic object is the fact that such an attempt at aesthetic analysis leads to distinctions and comparison of a more refined nature than those ordinarily made. His objects of study include polygonal forms, ornaments and tilings, vases, melody and harmony, and musical quality in poetry. Birkhoff's work is in the spirit of many a great artist and artisan of bygone days. The writer recalls the impression made upon him by finding Sheraton, the great creator of 18 th century furniture, starting a treatise on design with descriptive geometry and ending with sideboards.

In addition to this principal work Birkhoff wrote a number of papers on the subject, and spent a half year on leave of absence in the Far East and Europe to gather material. His study has been of interest to artists, musicians, psychologists, historians, and even mathematicians. The writer has found Birkhoff's theories on melody of practical value in the problem of effective improvisation on the organ. The high value which Birkhoff put on the aesthetic is illustrated by the answer he gave a professional musician as to why one should study mathematics. "One should study mathematics," said Birkhoff, "because it is only through mathematics that Nature can be conceived in harmonious form."

(f) Basic geometry. Birkhoff and Ralph Beatley joined forces in the interest of the teaching of elementary geometry around 1929. In 1932 Birkhoff [52] wrote a set of postulates for plane geometry based on the scale and protractor. These studies were capped by a textbook [53] on Basic geometry, written with Beatley and published in 1940. The necessity of undefined terms and assumptions is emphasized from the outset, doing away with "self-evident truths." There are five fundamental postulates, seven basic theorems and nineteen other theorems together with seven in loci. The system of real numbers is incorporated into three of the five axioms leading at once to the heart of geometry. Reports on the use of the book in practice are favorable. In any case the introduction of new ideas from the pen of a man as eminent as Birkhoff should be a great stimulus to the subject of elementary geometry.

In summarizing the mathematical work of Birkhoff a sentence of Poincaré comes to mind. Of the periodic orbits in dynamical systems he says that they are "la seule brèche par où nous puissons essayer de pénétrer dans une place jusqu'ici réputé inabordable." Poincaré would be amazed to see the extent to which Birkhoff has widened this breech and opened many others. His zeal never flagged. 
During the major part of his life Birkhoff was the acknowledged leader of American mathematics. His nomination as President of the International Congress of Mathematics that was to have been held at Cambridge, Massachusetts in 1940 was symbolic of this fact. Learned societies and universities the world over honored him. His work was crowned with four prizes. In the case of a man such as Birkhoff, a ranking at this time in the hierarchy of the great, in anticipation of the verdict of history, seems of doubtful value. The author can find no words adequate to define him except that he was profoundly unique. Those who knew him best can add very simply that he was a kind and courageous friend for whom they will have a lasting affection.

\section{BIBLIOGRAPHY}

The writer has had the benefit of a bibliography of Birkhoff's work prepared by Raymond C. Archibald, who is completing his work on this subject. Including abstracts, Birkhoff published approximately 190 papers, of which approximately one third were abstracts.

1. With H. S. Vandiver, On the integral divisors of $a^{n}-b^{n}$, Ann. of Math. vol. 5 (1904) pp. 173-180.

\section{Asymptotic expansions, Boundary values, Sturm-Liouville Series}

2. On the asymptotic character of the solutions of certain linear differential equations containing a parameter, Trans. Amer. Math. Soc. vol. 9 (1908) pp. 219-231.

3. Boundary value and expansion problems of ordinary linear differential equations, Trans. Amer. Math. Soc. vol. 9 (1908) pp. 373-395.

4. Existence and oscillation theorems for a certain boundary value problem, Trans. Amer. Math. Soc. vol. 10 (1909) pp. 259-270.

5. On the solutions of ordinary linear homogeneous differential equations of the third order, Ann. of Math. vol. 12 (1911) pp. 103-127.

6. Note on the expansion problems of ordinary linear differential equations, Rend. Circ. Mat. Palermo vol. 36 (1913) pp. 115-126.

7. A theorem on series of orthogonal functions with an application to Sturm-Liouville series, Proc. Nat. Acad. Sci. U.S.A. vol. 3 (1917) pp. 656-659.

8. With R. E. Langer, The boundary problems and developments associated with a system of ordinary linear differential equations of first order, Proceedings of the American Academy of Arts and Sciences vol. 58 (1923) pp. 49-128.

9. With M. R. Hestenes, Natural isoperimetric conditions in the calculus of variations, Duke Math. J. vol. 1 (1935) pp. 198-286.

II. LINEAR DIFFERENTIAL EQUATIONS AND GENERALIZED RIEMANN PROBLEM

10. Singular points of ordinary linear differential equations, Trans. Amer. Math. Soc. vol. 10 (1909) pp. 436-470.

11. A simplified treatment of the regular singular point, Trans. Amer. Math. Soc. vol. 11 (1910) pp. 199-202. 133.

12. A theorem on matrices of analytic functions, Math. Ann. vol. 74 (1913) pp. 122- 
13. The generalized Riemann problem for linear differential equations and the allied problems for linear difference and q-difference equations, Proceedings of the American Academy of Arts and Sciences vol. 49 (1913) pp. 521-568.

14. Infinite products of analytic matrices, Trans. Amer. Math. Soc. vol. 17 (1916) pp. 386-404.

\section{LINEAR DIFFERENCE EQUATIONS}

15. General theory of linear difference equations, Trans. Amer. Math. Soc. vol. 12 (1911) pp. 243-284.

16. Formal theory of irregular linear difference equations, Acta Math. vol. 54 (1930) pp. 205-246.

17. With W. J. Trjitzinsky, Analytic theory of singular difference equations, Acta Math. vol. 60 (1933) pp. 1-89.

\section{Dynamical systems}

18. Quelques theròmes sur le mouvement des systèmes dynamiques, Bull. Soc. Math. France vol. 40 (1912) pp. 305-323.

19. Proof of Poincare's geometric theorem, Trans. Amer. Math. Soc. vol. 14 (1913) pp. 14-22.

20. The restricted problem of three bodies, Rend. Circ. Mat. Palermo vol. 39 (1915) pp. 265-334. (Awarded the Quirini Stampalia Prize by the Royal Venice Institute of Science.)

21. Dynamical systems with two degrees of freedom, Trans. Amer. Math. Soc. vol. 18 (1917) pp. 199-300. (Awarded the first Bôcher Memorial Prize in 1923.)

22. Recent advances in dynamics, Science vol. 51 (1920) pp. 51-55.

23. Surface transformations and their dynamical applications, Acta Math. vol. 43 (1920) pp. 1-119.

24. An extension of Poincare's last geometric theorem, Acta Math. vol. 47 (1925) pp. 297-311.

25. Stability and the equations of dynamics, Amer. J. Math. vol. 49 (1927) pp. 1-38.

26. Dynamical systems, Amer. Math. Soc. Colloquium Publications, vol. 9, New York, 1927.

27. With P. A. Smith, Structure analysis of surface transformations, J. Math. Pures Appl. (9) vol. 7 (1928) pp. 345-379.

28. Une généralisation à n dimensions du dernier thêrème de gêométrie de Poincarê, C. R. Acad. Sci. Paris vol. 192 (1931) pp. 196-198.

29. Proof of a recurrence theorem for strongly transitive systems; Proof of the ergodic theorem, Proc. Nat. Acad. Sci. U.S.A. vol. 17 (1931) pp. 650-655; 656-660.

30. With B. O. Koopman, Recent contributions to the ergodic theory, Proc. Nat. Acad. Sci. U.S.A. vol. 18 (1932), pp. 279-282.

31. With D. C. Lewis, Jr., On the periodic motions near a given periodic motion of a dynamical system, Annali di Mat. Pura Appl. vol. 12 (1933) pp. 117-133.

32. Sur le problème restreint des trois corps (Premier Mémoire), Annali della R. Scuola Normale Superiore Pisa (2) vol. 4 (1935) pp. 267-306.

33. Nouvelles recherches sur les systèmes dynamiques, Mémoriae Pont. Acad. Sci. Novi Lyncaei vol. 1 (1935) pp. 85-216. (Crowned by the Pontifical Academy of Science.)

34. Sur le probleme restreint des trois corps (Second Mémoire), Annali della R. Scuola Normale Superiore Pisa (2) vol. 5 (1936) pp. 1-42.

35. Some unsolved problems of theoretical dynamics, Science vol. 94 (1941) pp. 598-600. 


\section{Physical theories}

36. Books on relativity, Bull. Amer. Math. Soc. vol. 28 (1922) pp. 215-221.

37. The origin, nature and influence of relativity (Lowell Institute Lectures), New York, 1925.

38. A theory of matter and electricity, Proc. Nat. Acad. Sci. U.S.A. vol. 13 (1927) pp. 160-165.

39. The hydrogen atom and the Balmer formula, Proc. Nat. Acad. Sci. U.S.A. vol. 13 (1927) pp. 165-169.

40. A mathematical critique of some physical theories, Bull. Amer. Math. Soc. vol. 33 (1927) pp. 165-181. (Awarded the annual prize of the American Association for the Advancement of Science.)

41. Some remarks concerning Schrödinger's wave equation, Proc. Nat. Acad. Sci. U.S.A. vol. 19 (1933) pp. 339-344. 325.

42. Electricity as a fluid, Journal of the Franklin Institute vol. 226 (1938) pp. 315-

43. Matter, electricity and gravitation in flat space-time, Proc. Nat. Acad. Sci. U.S.A. vol. 29 (1943) pp. 231-239.

44. The mathematical nature of physical theories, American Scientist vol. 31 (1943) pp. 281-310.

44a. El concepto matemático de tiempo y la gravitación, Boletin de la Sociedad Matemática Mexicana vol. 1 (1944) pp. 1-24.

\section{Miscellaneous WORKS}

45. With O. D. Kellogg, Invariant points in function space, Trans. Amer. Math. Soc. vol. 23 (1922) pp. 96-115.

46. A determinant formula for the number of ways of coloring a map, Ann. of Math. vol. 14 (1912) pp. 42-46.

47. The reducibility of maps, Amer. J. Math. vol. 35 (1913) pp. 115-128.

48. On the number of ways of coloring a map, Proc. Edinburgh Math. Soc. (2) vol. 2 (1930) pp. 83-91.

49. On the polynomial expressions for the number of ways of coloring a map, Annali della R. Scuola Normale Superiore Pisa (2) vol. 3 (1934) pp. 1-19.

50. General mean value and remainder theorems with applications to mechanical differentiation and quadrature, Trans. Amer. Math. Soc. vol. 7 (1906) pp. 107-136.

51. Aesthetic measure, Cambridge, 1933.

52. A set of postulates for plane geometry based on scale and protractor, Ann. of Math. vol. 33 (1932) pp. 329-345.

53. With Ralph Beatley, Basic geometry, New York, 1940.

54. Fifty years of American mathematics, Amer. Math. Soc. Semicentennial Publications, vol. 2, 1938, pp. 270-315. 